\title{
Semantic Relation between Words with the Web as Information Source
}

\author{
Tanmay Basu and C.A. Murthy \\ Machine Intelligence Unit, Indian Statistical Institute, Kolkata, India \\ \{tanmaybasu_r, murthy\}@isical.ac.in
}

\begin{abstract}
Semantic relation is an important concept of information science. Now a days it is widely used in semantic web. This paper aims to present a measure to automatically determine semantic relation between words using web as knowledge source. It explores whether two words are related or not even if they are dissimilar in meaning. The proposed formula is a function of frequency of occurrences of the words in each document in the corpus. This relationship measure will be useful to extract semantic information from the web . Experimental evaluation on ten manually selected word pairs using the WebKb data as information source demonstrates the effectiveness of the proposed semantic relation.
\end{abstract}

Keywords: semantic relation, page count.

\section{Introduction}

Web mining[2] has introduced a new era in the field of information science. It is the process of extracting knowledge such as patterns, relations from web data. The world wide web is a huge, diverse and dynamic information source. As the web contents grow, it becomes increasingly difficult to manage and classify its information. One of the most important applications of web mining is the web search engine. Semantic relation[1] is required to improve the query search results of the web search engine. It has several applications in fields such as natural language processing, text mining, semantic web [6] 7].

Semantic relation between two terms means how much two terms are related even if they are dissimilar in meaning. For example $c$ programming is a very frequent word pair in the web though they have no similarity in meaning. In this word pair $c$ is a letter and programming is a word in English language. Thus meaning wise $c$ and programming are very different. But, in the web they occur frequently. We intend to find this semantic relation between two words. The page count of $c$ programming in Google 1 is 2,090,0002 whereas the same for $u$ programming is 2,810 , where page count 4 of a word is the number of pages that contain the query words. This means that $u$ and programming are not semantically as much related as $c$ and programming.

\footnotetext{
1 http://www.google.com

2 This Google page count is taken on June 2009.

S. Chaudhury et al. (Eds.): PReMI 2009, LNCS 5909, pp. $267-2722009$.

(C) Springer-Verlag Berlin Heidelberg 2009
} 
This measure is suggested to determine semantic relation between two words using the web as information source. The frequency of the words in each document are measured rather than page count and normalize it over all documents. The other methods available in the literature are mainly based on page count of a word, such as Normalized Google Distance 3 . But page count is not sufficient to measure the semantic relation between two words. Page count provides the number of pages in which a word (or words) occurs. It does not indicate the number of times a word has occurred in each of this page. A word may appear many times in a document and once in the other document, but page count will ignore this.

This paper is described as follows. In section 2 we discuss our proposed semantic relation and its properties. Section 3 describes related work and comparison with the proposed measure whereas the last section describes the conclusions and future works.

\section{Proposed Measure of Semantic Relation}

Various semantic similarity measures are available in the literature. Different methods have been found to be useful in several applications. After extensively investigating a number of methods this measure is proposed to determine the semantic relation between two words which is applicable to any set of documents.

\subsection{Formulation}

Given two words $\mathrm{x}$ and $\mathrm{y}$, we need to find the semantic relation between them on the basis of a corpus(here web pages). The proposed semantic relation named as Semantic Word Distance(SWD) is defined as

$$
\operatorname{SWD}(\mathrm{x}, \mathrm{y})= \begin{cases}-1 & \text { if } f_{i}(x)=f_{i}(y)=0 \forall i \in \mathrm{M} \\ \left|\frac{\mathrm{wr}(\mathrm{x}, \mathrm{y})+\mathrm{wr}(\mathrm{y}, \mathrm{x})}{2}-1\right| & \text { otherwise }\end{cases}
$$

where $\mathrm{M}$ is the total number of pages from which we will find the relation between the words e.g. the number of pages indexed by a web search engine and Word Ratio(wr) is defined as,

$$
\mathrm{wr}(\mathrm{x}, \mathrm{y})=\frac{1}{M} \sum_{i=1}^{M} \max \left(I_{i}(x), I_{i}(y)\right) \frac{f_{i}(x)+1}{f_{i}(y)+1}
$$

Here $f_{i}(x)$ denotes the number of times the word $x$ occurs in the $i t h$ page and

$$
\mathrm{I}_{i}(x)= \begin{cases}0 & \text { if } f_{i}(x)=0 \\ 1 & \text { otherwise }\end{cases}
$$

That is we are neglecting the pages where none of the words occur. We are measuring the semantic relation between two words depending on the score of 
SWD. Note that if $f_{i}(x)=f_{i}(y) \neq 0 \forall i \in \mathrm{M}$ then $\operatorname{SWD}(\mathrm{x}, \mathrm{y})=0$ i.e. we can say the semantics of $\mathrm{x}$ and $\mathrm{y}$ are exactly same on a particular information source. $\mathrm{SWD}(\mathrm{x}, \mathrm{y})=0$ means the semantic relation between $\mathrm{x}$ and $\mathrm{y}$ is the strongest. As the value of SWD approaches zero, the relationship between the word pair becomes stronger. Essentially the above measure finds, for each page, the ratios of the number of occurrences of the word pair and averages it over the corpus. If the ratios are closer to one, the two words are more semantically related except for $f_{i}(x)=f_{i}(y)=0 \forall i$. This idea will be appropriate for the word pair like $c$ programming which exists simultaneously on the web pages but has no similarity in meaning.

\subsection{Properties}

Now we will discuss some properties of Semantic Word Distance.

- SWD is symmetric. For every pair of word $x, y$ we have $\operatorname{SWD}(x, y)=\operatorname{SWD}(y, x)$.

- The value of SWD must be greater than or equal to 0 except for the case of $f_{i}(x)=f_{i}(y)=0 \forall i$ and irrespective of the values of $\max \left(I_{i}(x), I_{i}(y)\right) \forall i$. As we know that $\mathrm{a}+\frac{1}{\mathrm{a}} \geq 2$ where $\mathrm{a} \in N$, a set of positive integers. Then we can state from equation (11) that $\operatorname{SWD}(\mathrm{x}, \mathrm{y})=\frac{1}{2}\left[\mathrm{wr}(\mathrm{x}, \mathrm{y})+\frac{1}{\operatorname{wr}(\mathrm{x}, \mathrm{y})}\right]-1 \geq 0$

- If $\operatorname{SWD}(\mathrm{x}, \mathrm{y})=0$ then we have

$$
\begin{gathered}
{\left[\frac{\mathrm{wr}(\mathrm{x}, \mathrm{y})+\mathrm{wr}(\mathrm{y}, \mathrm{x})}{2}\right]-1=0} \\
\text { i,e } \frac{1}{M} \sum_{i=1}^{M}\left\{\left(\frac{f_{i}(x)+1}{f_{i}(y)+1}+\frac{f_{i}(y)+1}{f_{i}(x)+1}\right) \max \left(I_{i}(x), I_{i}(y)\right)\right\}=2
\end{gathered}
$$

If $\max \left(I_{i}(x), I_{i}(y)\right)=1 \forall i$ then $f_{i}(x)+1=f_{i}(y)+1 \forall i$ as we know that $a+\frac{1}{\mathrm{a}}=2$ only when $\mathrm{a}=1$. But it is not necessarily true that $\max \left(I_{i}(x), I_{i}(y)\right)=1 \forall i$. Therefore $\operatorname{SWD}(\mathrm{x}, \mathrm{y})=0$ does not imply $f_{i}(x)=f_{i}(y) \forall i=1,2, \ldots, M$.

-So SWD is not a metric. Also it need not satisfy the triangular inequality i.e. $\operatorname{SWD}(\mathrm{x}, \mathrm{y})+\operatorname{SWD}(\mathrm{y}, \mathrm{z}) \geq \operatorname{SWD}(\mathrm{x}, \mathrm{z}) \forall x, y, z$ need not be true.

Choose $f_{i}(x)+1=X_{i}, f_{i}(y)+1=Y_{i}, f_{i}(z)+1=Z_{i}$ and $\max \left(I_{i}(x), I_{i}(y)\right)=$ $1 \forall i$. Therefore the triangular inequality becomes

$$
\begin{gathered}
\frac{1}{2 M} \sum_{i=1}^{M}\left(\frac{X_{i}}{Y_{i}}+\frac{Y_{i}}{X_{i}}\right)-1+\frac{1}{2 M} \sum_{i=1}^{M}\left(\frac{Y_{i}}{Z_{i}}+\frac{Z_{i}}{Y_{i}}\right)-1 \geq \frac{1}{2 M} \sum_{i=1}^{M}\left(\frac{X_{i}}{Z_{i}}+\frac{Z_{i}}{X_{i}}\right)-1 \\
i, e \sum_{i=1}^{M}\left(\frac{X_{i}}{Y_{i}}+\frac{Y_{i}}{X_{i}}+\frac{Y_{i}}{Z_{i}}+\frac{Z_{i}}{Y_{i}}-\frac{X_{i}}{Z_{i}}-\frac{Z_{i}}{X_{i}}\right)-2 M \geq 0
\end{gathered}
$$

Let $X_{i} \leq Y_{i} \leq Z_{i} \forall i$ and $Y_{i}=p X_{i}$ and $Z_{i}=q X_{i}$ where $\mathrm{p} \geq 3$ and $\mathrm{q}=2 \mathrm{p}$. This violates the condition of inequality (2). Hence swd is not a metric. 


\subsection{Similarity Measurement}

The lower limit of SWD is -1 which indicates, this is beyond the scope of SWD to determine the relation between the words on a particular corpus. This occurs only when no two words appear in any of the documents of the corpus. In the other cases SWD always provides non negative values. If the SWD value is 0 then it indicates that the semantic relation between the word pair is strongest. It has no upper limit. The relationship between the words decreases as long as the value grows from 0 . For simplicity we assume 2 as the upper limit. If two words appear in 1:11 ratio or more than that on an average, then SWD value of two words is greater than 2 and we will just ignore it i.e. two words are dissimilar when their SWD value is greater than 2.

\section{Comparison with Other Measures}

Semantic similarity measures have proved their worth in web related tasks. A number of methods on semantic relation have been proposed in the last few years. Some of them are subjective i.e. based on some specific corpus e.g. Wordnet ${ }^{3}$ 9] or Wikipedia 8. No measure based on a specific corpus is considered here for comparison. Some of the corpus independent measures are WebJaccard, WebOverlap, WebDice, WebPMI [4], Normalized Google Distance(NGD) 3]. WebJaccard, WebOverlap, WebDice, WebPMI are the modifications of the popular co-occurrence measures Jaccard, Overlap, Dice and PMI respectively. NGD is also a popular measure and is not confined to a particular web search engine. The range of WebJaccard, WebDice and WebOverlap are in between 0 and 1 . According to these three formulae two words are similar if the value is 1 and the relationship decreases when the value decreases to 0. But these three measures do not incorporate the total number of pages of the corpus(for example total number of pages indexed by the search engine) which is very important in measuring the semantic relation. NGD normally takes the values between 0 and 1 though the value lies in between 0 and $\infty[3$. For NGD two words are similar if the value is 0 and the relationship decreases as it grows to 1 .

All these measures are mainly based on page count. Page count of a word may not provide the full notion of the word e,g Google returns $12,700,000^{4}$ as the page count for electronic AND calculator whereas 131,000,000 for electronic AND food although electronic is strongly related to calculator than food. In SWD the frequency of a word in a document is measured rather than page count and normalize it over the corpus. Hence SWD has more proficiency in finding the relation. For example if one word occurs for 45, 30, 22, 16, 53 times in five documents and other word occurs for 2, 3, 4, 1,6 times in the same five documents, SWD will produce a result different from zero i,e semantically the words are not that much related. But page count based methods generally produce a result which indicate that the words are close semantically. This is

${ }^{3}$ http://wordnet.princeton.edu

4 This count is taken on May 2009. 
the main difference between the proposed definition and the page count based definition.

The performance of the proposed Semantic Word Distance has been evaluated and compared with the other methods mentioned earlier. In the next two section we will discuss about the data sets used and the methods of evaluation.

\subsection{The Data Source}

Ten word pairs are selected manually to measure semantic relation between each of them. For this the WebKb data set [5] is used. This data set contains WWW pages collected from computer science departments of various universities in January 1997 by the World Wide Knowledge Base (WebKb) project of the CMU text learning group. The 8,282 pages were manually classified into seven categories. For each category the data set contains pages from the four universities Cornell (867), Texas (827), Washington (1205) and Wisconsin (1263).

\subsection{Evaluation}

Here WebKb data set is used as the information source. Basically it will be determined, how much two words are related depending on this corpus. It may again be mentioned here, that two words are more related if the SWD, NGD, WebPMI values are close to zero and the WebJaccard, WebOverlap, WebDice values are close to one.

It is observed from Table 1 that SWD is seemingly better than NGD and WebPMI for almost all the ten word pairs. Research and paper are very related words as WebKb is a university data set. SWD determines this word pair is very related whereas the other measures fail to show this. They have shown research and paper are related but not so much as it is shown by SWD. Again the word pairs university-graduate, web-page, programming-assignment and coursecoordinator have the higher NGD and WebPMI values though they are very

Table 1. Performance of Semantic Relation Between the Selected Word Pairs

\begin{tabular}{|c|c|c|c|c|c|c|}
\hline $\begin{array}{c}\text { Selected } \\
\text { Word Pair }\end{array}$ & $\begin{array}{c}\text { Web } \\
\text { Jaccard }\end{array}$ & $\begin{array}{c}\text { Web } \\
\text { Overlap }\end{array}$ & $\begin{array}{c}\text { Web } \\
\text { Dice }\end{array}$ & $\begin{array}{c}\text { Web } \\
\text { PMI }\end{array}$ & NGD & $\begin{array}{c}\text { SWD } \\
\text { (Proposed) }\end{array}$ \\
\hline research - paper & 0.250 & 0.641 & 0.400 & 0.651 & 0.732 & 0.168 \\
\hline university - graduate & 0.271 & 0.776 & 0.426 & 0.698 & 0.716 & 0.129 \\
\hline web - page & 0.281 & 0.813 & 0.439 & 0.487 & 0.780 & 0.001 \\
\hline programming - assignment & 0.202 & 0.625 & 0.336 & 0.716 & 0.747 & 0.030 \\
\hline course-coordinator & 0.041 & 0.417 & 0.080 & 0.678 & 0.868 & 0.429 \\
\hline computer - science & 0.760 & 0.975 & 0.864 & 0.598 & 0.379 & 0.076 \\
\hline home - page & 0.676 & 0.899 & 0.806 & 0.632 & 0.415 & 0.171 \\
\hline sound - vehicle & 0.021 & 0.064 & 0.041 & 1.694 & 0.748 & 0.955 \\
\hline book - culture & 0.031 & 0.315 & 0.061 & 1.629 & 0.749 & 0.818 \\
\hline speaker - player & 0.018 & 0.038 & 0.037 & 1.924 & 0.714 & 0.908 \\
\hline
\end{tabular}


related (which is determined by SWD with a lower value). This is because the co-existence of these three word pairs in each document of the corpus is very low. The word pairs sound-vehicle, book-culture and speaker-player are weakly related which is shown by SWD like the other measures according to the general understanding of the meaning of the words and their interrelations. It may be noted that WebJaccard, WebDice and WebOverlap are not measuring the total number of pages. Even then the proposed measure is giving better results except for the word pairs computer-science and home-page. For these two word pairs WebOverlap and WebDice are giving slightly better results than SWD because the co-existence of these word pairs are very high and those measures do not count the total number of pages.

\section{Conclusion}

A method is suggested to determine semantic relation between two words. This is not based on the traditional page count method. The method counts the number of occurrences of each word in each document and normalizes it over the corpus. By this the entire knowledge of a word pair in a document can be obtained without measuring the co-existence of the words. The co-existence of two words is automatically measured in the formula. The main application of SWD is to measure the semantic relation between words using the web search engine for better query search results. In future we will apply SWD in web page classification and to measure the similarity between web pages.

\section{References}

1. Gracia, J.L., Mena, E.: Web Based Measure of Semantic Relatedness. In: Bailey, J., Maier, D., Schewe, K.-D., Thalheim, B., Wang, X.S. (eds.) WISE 2008. LNCS, vol. 5175, pp. 136-150. Springer, Heidelberg (2008)

2. Kosala, R., Blockeel, H.: Web Mining Research: A Survey. ACM SIGKDD Explorations 2, 1-15 (2000)

3. Cilibrasi, R.L., Vitanyi, P.M.: The Google similarity distance. IEEE Transactions on Knowledge and Data Engineering 19(3), 370-383 (2007)

4. Bollegala, D., Matsuo, Y., Ishizuka, M.: Measuring Semantic Similarity Between Words Using Web Search Engines. In: Proceedings of WWW 2007, Banff, Canada (2007)

5. http://www.cs.cmu.edu/afs/cs.cmu.edu/project/theo-20/www/data/ webkbdata.gtar.gz

6. Berners-Lee, T., Hendler, J., Lassila, O.: The Semantic Web. Scientific American, 34-43 (May 2001)

7. Catherine, M.C., Frank, S.M.: Which Semantic Web. In: Proceedings of ACM Hypertext, pp. 57-66 (2003)

8. Strube, M., Ponzetto, S.P.: Wikirelate! Computing Semantic Relatedness Using Wikipedia. In: AAAI. AAAI Press, Menlo Park (2006)

9. Pedersen, T., Banerjee, S.P.: Maximizing Semantic Relatedness to Perform Word Sense Disambiguation. Research Report. UMSI 2005 (2005) 